\title{
DIVERSIDADE E DISTRIBUIÇĀO GEOGRĀFICA DAS ESPÉCIES DE ARISTIDA L. (GRAMINEAE) OCORRENTES NO BRASIL
}

\author{
Hilda Maria Longhi-Wagner ${ }^{1}$
}

RESUMO - Trinta e quatro espécies de Aristida L. foram confirmadas até agora para o Brasil, 13 das quais exclusivas desta área. A maior parte das mesmas está relacionada a dois padrões gerais de distribuição geográfica: o tropical e o subtropical. Ambos incluem tanto espécies de ampla distribuição quanto restritas a determinadas áreas do Brasil.

São analisados 7 caracteres taxonômicos, correlacionando-os à distribuição das espécies: tipo de inflorescência; comprimento relativo das glumas; e os seguintes relativos ao lema: coluna, calo, articulação, sulco ventral e aristas. De um modo geral, as espécies do centro e norte do Brasil apresentam uma descontinuidade morfológica mais acentuada do que as da Região Sul, podendo mais facilmente serem diferenciadas no campo.

$\widehat{E}$ apresentada uma lista das espécies confirmadas, sua distribuição geográfica e uma discussão sobre a importância dos 7 caracteres taxonômicos mencionados acima, os quais são ilustrados.

Palavras-chave: Diversidade, distribuição geográfica, Gramineae, Aristida.

\begin{abstract}
Thirty four species of Aristida L. were confirmed so far for Brazil, 13 of them exclusives of this area. Most are related to two general patterns of geographical distribution: the tropical and the subtropical. Both include species with a broad distribution and species with a limited distribution.

Seven taxonomic characters are analysed and correlated to the species distribution: type of inflorescence; relative lenght of the glumes; and lemma: column, callus, articulation, ventral groove and awns.

As a general rule, the species which occur in Northern and Central Brazil present a more remarkable morphological discontinuity than the species from the South, and can more easily be differentiated in the field.
\end{abstract}

1 - Departamento de Botânica, Universidade Federal do Rio Grande do Sul. Bolsista de Pesquisa do CNPq. 
A list of the species, their geographical distribution and a discussion about the 7 taxonomic characters mentioned above, including illustrations of the latter, are presented.

Key-words: Diversity, geographical distribution, Gramineae, Aristida.

\section{Introdução}

O gênero Aristida $L$. foi descrito com base na espécie cosmopolita A. adscensionis L. e inclui cerca de 280 espécies. Os trabalhos mais abrangentes sobre o gênero são os de Trinius \& Ruprecht (1842) e a monografia de Henrard (1929, 1932), esta última reunindo parte dos dados já discutidos pelo autor em trabalhos prévios (Henrard, 1926, 1927, 1928).

Para o Brasil, especificamente, 11 espécies foram tratadas por Nees (1829), sob Chaetaria, e 18 por Doell (1878). Após estes dois trabalhos o gênero foi tratado em floras regionais por Smith et al. (1981), Severo (1982), Renvoize (1984, 1988) e Longhi-Wagner (1986), não existindo entretanto qualquer chave que reúna todas as espécies ocorrentes nas diferentes regiões do país.

Henrard $(1929,1932)$ citou 36 espécies para o Brasil, este número coincidindo aproximadamente com os dados que foram levantados até o momento pelo presente trabalho, onde 34 espécies foram confirmadas, porém não havendo esta mesma coincidência em relação aos táxons aceitos. Isto é devido especialmente à sinonimização de algumas espécies consideradas independentes por Henrard $(1929,1932)$, como por exemplo $A$ setifolia e $A$. doelliana, $A$. torta e $A$. tincta, $A$. marginalis e $A$. gibbosa, entre outras, e também à descrição de novas espécies, como $A$. riograndensis e $A$. pendula.

A característica principal do gênero Aristida, que o torna de fácil reconhecimento no campo, é a presença de espiguetas unifloras associadas a lemas triaristados. Entretanto, na seção Streptachne (R. Br.) Dom., sem representantes no Brasil, ocorrem espécies com aristas laterais muito reduzidas até ausentes, e $A$. parvula (Nees) De Winter, única espécie (sul-africana) da seção Schizachne (Trin. \& Rupr.) De Winter, apresenta uma artista simples e bigeniculada, o que De Winter (1965) considera um estado secundário do caráter dentro do gênero; a mencionada espécie apresenta anatomia foliar característica de Aristida. Tendência à redução das artistas laterais pode também ser observada em $A$. torta, pertencente à seção Aristida e comum no Centro e Norte do Brasil.

Considerando as gramíneas como um todo, a combinação de espiguetas com um só antécio e lemas triaristados pode representar uma autapomorfia para a tribo Aristideae, tal como aceita por De Winter (1965) e Clayton \& Renvoize (1986), que inclui três gêneros: Aristida, Stipagrostis Nees, com cerca de 38 espécies africanas de aristas plumosas e Sartidia, com quatro espécies da Āfrica e Āsia Central. 
Dentro da tribo Aristideae, assim como entre as demais gramíneas que apresentam a "síndrome Kranz", Aristida destaca-se por ser o único gênero até agora detectado que apresenta uma dupla bainha parenquimática com cloroplastídeos especializados, referido por Brown (1977) como tipo "DS" (double sheath); os demais membros da subfamília Chloridoideae, incluindo Stipagrostis e excluindo Sartidia que é "não-Kranz", pertencem ao tipo PS (parenchyma sheath) de Brown (1977). Este caráter, portanto, pode ser considerado uma autapomorfia para o gênero Aristida, tal como ele é aceito atualmente.

\section{Resultados e discussão}

\section{Taxonomia do gênero}

Devido especialmente às características do lema e à anatomia foliar peculiar, o gênero Aristida tem tido sua posição taxonômica discutida, tendo já sido incluído em diferentes subfamílias como Arundinoideae (Tateoka, 1957; Stebbins \& Crampton, 1961; Clayton \& Renvoize, 1986); Aristidoideae (Caro, 1982) e em Chloridoideae, esta última sendo a posição aceita pela maior parte dos autores, como Burkart (1969), Parodi (1961), Valls (1980) e Burman (1985).

É curioso notar que, nos sistemas mais antigos de classificação de gramíneas, como em Humboldt, Bonpland \& Kunth (1815), Bentham \& Hooker (1880), também nos trabalhos de Nees (1829), Doell (1878) e Henrard (1929) e, surpreendentemente, no trabalho mais recente de Smith et al. (1981), Aristida aparece sempre associado ao gênero Stipa, com o qual apresenta semelhanças na estrutura do lema. Porém, os dois gêneros apresentam claras diferenças anatômicas, devendo ser posicionados em subfamílias diferentes. Quanto à divisão infragenérica, 7 seções foram aceitas por Henrard (1929), de acordo com a estrutura do lema: Chaetaria, Pseudochaetaria, Streptachne, Arthratherum, Pseudarthratherum, Stipagrostis e Schistachne.

De Winter (1965), utilizando dados morfológicos, anatômicos e cariológicos, considerou Stipagrostis como um gênero válido e Schistachne como uma seção do mesmo. Com isto, aceita 5 das 7 seções consideradas por Henrard (1929) e inclui a seção monotípica Schizachne. Estas seções podem ser assim caracterizadas: Aristida (Chaetaria): lemas sem articulação, com ou sem coluna, três aristas desenvolvidas - cerca de 200 espécies de regióes tropicais e subtropicais de ambos os hemisférios; Pseudochaetaria: lemas articulados no ápice, sem coluna, três aristas desenvolvidas - três espécies sul-africanas; Arthratherum: lemas articulados na base de coluna, três aristas desenvolvidas cerca de 40 espécies; Pseudarthratherum: lemas articulados no ápice da coluna, três aristas desenvolvidas - cerca de 20 espécies, a maior parte na África, 5 em Galápagos e 1 no Brasil; Streptachne: lemas sem articulação, aristas laterais muito reduzidas até ausentes - cerca de 14 espécies, a maior parte 
da América do Norte meridional e um menor número na Austrália, Āfrica e América do Sul; Schizachne: lemas com arista simples, bigeniculada - uma só espécie, africana.

No Brasil, estão representadas as seções Aristida e Pseudarthratherum, esta última apenas com $A$. setifolia H. B. K.

\section{Espécies até o momento confirmadas para o Brasil}

A. adscensionis L., A. capillacea Lam., A. chapadensis Trin. \& Rupr., A. circinalis Lindm., A condylifolia Caro, A. echinulata Roseng. \& Izag., A. ekmaniana Henr., $A$ elliptica (Nees) Kunth, $A$. filifolia (Arech.) Herter, $A$. flaccida Trin. \& Rupr., A gibbosa Henr., A. glaziovii Henr., A jubata (Arech.) Herter, $A$. kleinii L. B. Smith, A. laevis (Nees) Kunth, $A$. longifolia Trin., $A$ megapotamica Spr. var. megapotamica e var. brevipes Henr., A. murina Cav., A. oligospira (Hack.) Henr., A. pendula Longhi-Wagner, $A$. recurvata H. B. $\mathrm{K}$., A riograndensis Severo \& Boldrini, A. riparia Trin. \& Rupr., A. sanctaeluciae Trin., A. setifolia H. B. K., A. spegazzinii Arech., A. succedanea Henr., A. teretifolia Arech., A. torta (Nees) Kunth, A. trinii Henr., A. uruguayensis Henr. var. uruguayensis e var. laevis Caro, $A$. venustula Arech., $A$. venustuloides Caro e Aristida sp. (A. trinii sensu Severo).

\section{Análise de caracteres de importância taxonômica no gênero e sua relação com a distribuição das espécies brasileịas}

Os caracteres que têm sido utilizados na taxonomia do gênero Aristida são relacionados principalmente com a estrutura da espigueta, destacando-se os do lema. Entre eles:

1. Comprimento relativo das glumas: a maior parte das espécies ocorrentes no Brasil apresenta glumas inferiores menores do que as superiores, o que é o estado mais comum no gênero e nas gramíneas de modo geral (Figura 2). Oito espécies apresentam o que Henrard (1929) chamou de posição inversa das glumas, ou seja, glumas inferiores maiores do que as superiores (Figura 3), e 5 espécies são variáveis em relação a este caráter, variação esta que pode ser verificada em uma mesma inflorescência.

Henrard (1932) utiliza este caráter em chave para separar grandes agrupamentos de espécies, o que muitas vezes dificulta a identificação daquelas que apresentam variação. $\overline{\mathrm{E}}$ interessante, por isto, serem analisadas várias espiguetas de uma mesma panícula.

2. Presença ou ausência de coluna na arista do lema: característica de grande importância que serve para separar grupos de espécies. É um caráter constante podendo ocorrer, naquelas espécies com coluna, variação no comprimento da mesma, embora dentro de limites pequenos.

Dezenove espécies brasileiras não apresentam coluna (Figuras 2-5), qua- 
torze das quais concentram-se na Região Sul. No Centro e Norte do Brasil, predominam as espécies com coluna no lema (Figuras 6-8).

De acordo com De Winter (1965), o desenvolvimento de uma coluna pelo alongamento e torção do ápice do lema, bem como a aquisição de uma zona de articulação, devem ser vistos como passos progressivos na evolução do grupo.

3. Calo do lema: o calo, antopódio de acordo com Caro (1961) e Severo (1982), é um prolongamento da porção basal do lema, podendo às vezes estar envolvida uma porção da ráquila (Clifford 1987), e apresenta-se coberto por pêlos antrorsos de densidade variável.

Esta característica, assim como a coluna e aristas dos lemas, está diretamente associada com a dispersão e estabelecimento do diásporo, em Aristida. .

O tipo de calo, se obtuso (Figura 6) ou agudo (Figura 7) está relacionado com o seu menor ou maior grau de desenvolvimento, respectivamente. Entre as espécies do Centro e Norte do Brasil, é um caráter muito bem definido, servindo para separá-las facilmente; diminui de importância, entretanto, nas espécies da Região Sul, pois a maior parte apresenta calo semelhante, que inclusive é difícil definir por ter um grau de desenvolvimento intermediário, sendo descrito como subagudo por Severo (1982), para várias espécies.

$\mathrm{O}$ calo freqüentemente apresenta um prolongamento apical glabro, o qual é tipicamente bífido em $A$. riparia (Figura 9). Em $A$. megapotamica, este prolongamento glabro freqüentemente é desviado para um lado, parecendo às vezes um calo bífido, com um dos "dentes” menos desenvolvidos (Figura 10).

Este caráter, embora importante na taxonomia do gênero, é utilizado a um nível mais baixo que o anterior, separando grupos menores de espécies.

4. Presença de articulação no lema: conforme já mencionado, os representantes das seções Pseudochaetaria, Arthratherum e Pseudarthratherum apresentam articulação localizada em diferentes pontos do lema, o que vai influir no tipo de diásporo da espécie.

Entre as espécies brasileiras, este caráter ocorre em $A$. setifolia, com limite sul de distribuição na região de São Paulo, na qual há um característico nódulo de articulação no ápice da coluna, separando facilmente astrês aristas (Figura 7). A. doelliana Henr., citada como uma segùnda espécie da seção Pseudarthratherum presente no Brasil, por Henrard (1929) e De Winter (1965), é aqui considerada sinônimo de $A$. setifolia .

Em A. trinii sensu Severo (1982), que difere por vários caracteres da verddeira $A$. trinii, tratando-se provavelmente de uma nova espécie, ocorre uma zona de articulação na base da coluna. É interessante salientar que este caráter relaciona este táxon com a seção Arthratherum, da qual não são conhecidos representantes na América do Sul, relacionamento este que necessita ser analisado com mais detalhe.

5. Comprimento das aristas dos lemas: dois tipos de características são utilizadas: o comprimento propriamente dito e a relação entre o comprimento das aristas laterais e a central. 
No gênero Aristida, as três aristas são geralmente subiguais. Neste caso, é utilizado o comprimento por si só, para a separação de determinadas espécies (Figuras 3, 6, 8).

Entre as espécies ocorrentes no Brasil, apenas $A$. flaccida e A. longifolia (Figura 2) apresentam aristas laterais nitidamente menores do que a central. Em A. torta, este caráter varia, desde aristas laterais $2 / 3$ da central até $1 / 3$ ou menos do comprimento da central (Figura 4). A. flaccida ocorre no sul do Brasil e as outras duas espécies, tem seu limite meridional de distribuição na região de São Paulo.

6. Tipo de inflorescência: a inflorescência, em Aristida, é uma panícula que varia desde laxa e pauciflora, até espiciforme e densiflora.

Henrard (1927) referiu-se ao grupo "ripariae", caracterizado pela panícula espiciforme densiflora associada à coluna do lema desenvolvida: $A$. megapotamica, $A$. oligospira, $A$. riparia e $A$. trinii (Figura 11). Na verdade, $A$. trinii deve ser excluída deste grupo, pois apresenta panícula subespeciforme bastante diferenciada (Figura 12). As outras três espécies separaram-se pelo tipo de calo do lema, agudo e freqüentemente desviado para um lado na primeira, obtuso na segunda e com prolongamento apical glabro e bífido na terceira. No Rio Grande do Sul, ocorre mais uma espécie com panícula espiciforme densiflora, $A$. trinii sensu Severo (non $A$. trinii Henr.), que apresenta coluna menos desenvolvida do que as espécies mencionadas acima.

A. elliptica apresenta um tipo exclusivo de inflorescência, com as espiguetas congestas no ápice dos colmos floríferos (Figura 13). Esta espécie é muito comum no Nordeste do Brasil, com limite sul na Bahia.

Panícula laxa e pauciflora, com poucas espiguetas concentradas no ápice dos ramos, ocorre em $A$. flaccida e em $A$. longifolia (Figura 14). Na primeira, o pedúnculo da inflorescência quebra na maturação, e esta é levada pelo vento, constituindo-se no diásporo da espécie. A. pendula e $A$. chapadensis também apresentam panícula laxa, porém subdensiflora (Figura 15).

A. murina, espécie que ocorre na Argentina e Uruguai penetrando no Brasil somente na região Sudoeste do Rio Grande do Sul, apresenta uma inflorescência flabeliforme exclusiva, com espiguetas mais concentradas no ápice dos colmos floríferos, mas não congestas (Figura 16).

As demais espécies brasileiras de Aristida não apresentam um padrão tão definido de inflorescência como as mencionadas acima, variando de aberta, contraída a subespiciforme.

7. Presença de sulco ventral no lema: os lemas, na maior parte das espécies ocorrentes no Brasil, são cilíndricos, com os bordos sobrepostos. Há um pequeno grupo de espécies, entretanto, onde os bordos são involutos, formando um sulco longitudinal ventral (Figura 5).

Entre as espécies com distribuição no Centro e Norte do Brasil, A. gibbosa, $A$. pendula e $A$. recurvata apresentam lemas sulcados, porém com sulcos menos pronunciados do que em $A$. circinalis, A. kleinii, A. spegazzinii e $A$. 
succedanea, que ocorrem no Sul e Sudeste do Brasil. Nestas quatro últimas espécies, este caráter está associado à posição nitidamente inversa das glumas, enquanto nas outras três, a relação entre o comprimento das 2 glumas é variável, embora predomine a posição inversa.

\section{Ciclo de vida}

A maior parte das espécies que ocorrem no Brasil são perenes. Só no Sudoeste ocorrem duas espécies anuais muito relacionadas, $A$. capillacea e $A$. sanctae-luciae. Ao contrário do que foi verificado por De Winter (1965) para a Âfrica do Sul, onde o grupo anual é composto de espécies sem coluna no lema, as duas espécies têm coluna, embora pouco desenvolvida. Outra espécie anual é A. adscensionis L., esta sim sem coluna no lema; esta espécie é citada como cosmopolita, porém no Brasil ocorre na Região Nordeste até a Bahia.

A. setifolia foi considerada espécie anual por Doell (1878), Henrard (1929) e Renvoize (1984). Entretanto, Henrard (1928) a havia descrito como perene. De acordo com observações feitas no campo para este trabalho, parece ser realmente perene.

\section{Habitat}

A presença quase universal da "Síndrome Kranz" em Chloridoideae, está relacionada com os habitats de alta luminosidade, temperatura elevada e "stress" hídrico aos quais espécies desta subfamília estão geralmente associadas, ocupando especialmente regiões tropicais e subtropicais. Da mesma forma, as espécies de Aristida, no Brasil e em outras áreas, ocorrem geralmente em solos pedregosos e secos, com exceção de $A$. capillacea, que muitas vezes é encontrada em solos brejosos, e A sanctae-luciae que pode ocorrer em ambiente semelhante, embora menos comumente.

As espécies de Aristida que ocorrem em zonas subtropicais até temperadas, com estações do ano definidas, mantêm o seu caráter megatérmico, uma vez que são de ciclo estival, e ocorrem associadas a solos secos e pedregosos.

\section{Distribuição geográfica}

A distribuição geral do gênero é apresentada na Figura 1.

A seção Aristida apresenta a mais ampla distribuição, ocorrendo em todo o hemisfério sul e no sul dos Estados Unidos, com uma zona de alta concentração de espécies na América do Sul, especialmente no Brasil.

Burkart (1975) menciona três principais domínios de gramíneas na América do Sul: o tropical e subtropical, formado por grupos megatérmicos e que 


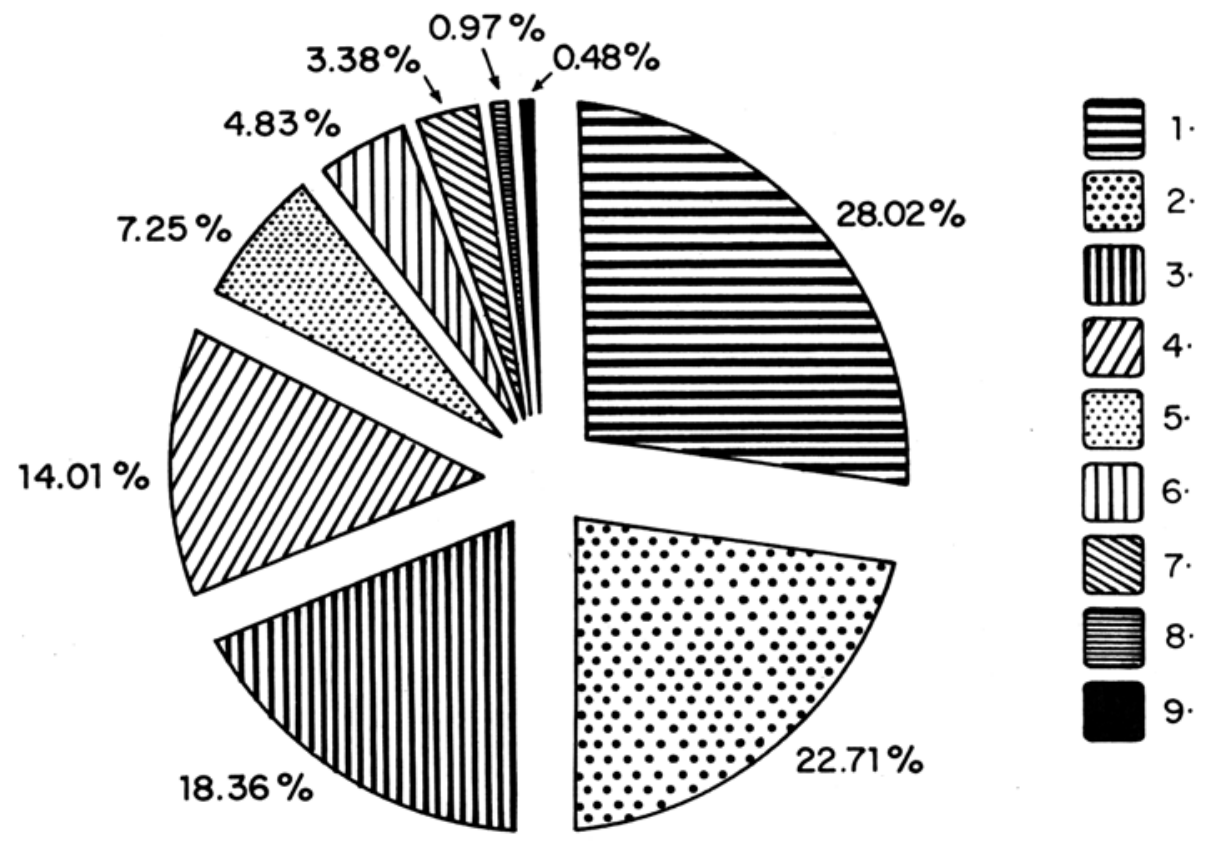

Figura 1 - Distribuição de Aristida: percentagem de espécies por área - 1. América do Sul; e Central; 2. América do Norte; 3. Āfrica; 4. Austrália; 5. Antilhas; 6. Āsia; 7. Madagascar; 8. Nova Caledônea; 9. Europa.

inclui os campos cerrados do Brasil Central, o norte do Brasil e a parte setentrional dos campos do sul do Brasil; o temperado, com grupos mesotérmicos que ocupam o "Pampa" da Argentina e os campos do Uruguai e Rio Grande do Sul meridional; as regiões mais frias, onde dominam as gramíneas microtérmicas. $\mathrm{O}$ gênero Aristida é incluído pelo autor nos dois primeiros grupos.

\section{Distribuição das espécies no Brasil}

A maior parte das espécies ocorrentes no Brasil pode ser enquadrada em dois padrões gerais de distribuição, o tropical e o subtropical.

\section{Distribuição tropical:}

Corresponde, em parte, aos campos tropicais e subtropicais (grupos megatérmicos) aceitos por Burkart (1975), sendo menos abrangente.

1.1. - Espécies que ocorrem desde a América Central e/ou México até aproximadamente a latitude do Trópico de Capricórnio: $A$. capillacea, $A$. longifolia, 
A. riparia, $A$. setifolia e $A$. torta . Aqui também pode ser incluída $A$. recurvata H. B. K., que atinge a latitude de $25^{\circ} \mathrm{S}$, diminuindo em abundância em direção sul, e que não foi detectada na Amazônia (Figura 17).

A. riparia foi citada para o Paraná por Ekman (1913) e Angely (1965), porém não teve sua ocorrência confirmada por Renvoize (1988) e nem no presente trabalho.

Este padrão de distribuição é semelhante ao padrão 1 referido por Giulietti \& Pirani (1988) e exemplificado por espécies de diferentes famílias.

1.2 - Espécie ocorrente no Paraguai e Brasil:

- A. oligospira ocorre na chamada Província Central do Brasil (Fernandes \& Bezerra 1990), além do Paraguai (Figura 19).

1.3 - Espécies exclusivas do Brasil:

a. Espécies que ocorrem desde o Nordeste ou Norte do Brasil até o sul da Bahia ou Minas Gerais: o primeiro caso refere-se à $A$. elliptica, muito comum na Região Nordeste, e o segundo à $A$. gibbosa, mais comum no norte e Brasil Central (Figura 21).

b. Espécies que ocorrem na Província Central e Bahia até a região sudeste: A. glaziovii, $A$. sanctae-luciae e $A$. pendula.

$A$. megapotamica var. brevipes está relacionada com este padrão de distribuição, porém estende-se até o Paraná (Figura 20).

A. pendula apresenta um padrão de distribuição que abrange serras da Cadeia do Espinhaço e de Goiás, já referido por Giulietti \& Pirani (1988). Segundo estes autores, este padrão evidencia uma provável ligação mais antiga entre as floras destas regiões que possuem características geológicas e climáticas semelhantes.

c. Espécie ocorrente em Minas Gerais, especialmente nos campos rupestres da Cadeia do Espinhaço, e em São Paulo: $A$. trinii (Figura 20). Esta espécie foi citada por Severo (1982) para o Rio Grande do Sul, porém o material sul-rio-grandense que serviu de base a esta citação pertence a outra espécie, provavelmente nova para a ciência, restrita ao sudoeste do Rio Grande do Sul, ocorrendo geralmente associada a afloramentos basálticos.

d. Espécies com distribuição restrita: $A$. chapadensis, só foi coletada, até o momento, na Serra da Chapada, em Mato Grosso e A. succedanea em São Paulo (Figura 21).

\section{Distribuição subtropical ou predominantemente tropical.}

Corresponde aproximadamente ao grupo temperado aceito por Burkart (1975), porém com uma abrangência mais ampla, pois inclui todo o sul do Brasil, diminuindo sua expressão na vegetação em direção norte. 
2.1 - Espécies que ocorrem no Uruguai e/ou Argentina e que penetram pelo extremo sul do Brasil:

Parte destas espécies tem seu limite setentrional de ocorrência na região do Rio Grande do Sul, enquanto um número mais reduzido atinge menores latitudes. No primeiro caso encontram-se $A$. condylifolia, $A$. echinulata, $A$. murina, $A$. uruguayensis e $A$. venustoloides. No segundo caso, são incluídas espécies que diminuem em abundância em direção norte: $A$. spegazzinii e $A$. filifolia (Figuras 18 e 19). A primeira espécie é pouco comum no sul do Brasil; da segunda espécie, foi analisada apenas uma coleta procedente de São Paulo; não foi citada por Smith et al. (1981) e Renvoize (1988) para Santa Catarina e Paraná, respectivamente, onde é provável sua ocorrência.

A. murina e $A$. spegazzinii foram referidas para a flora Patagônica por Nicora (1978), sendo as duas espécies deste grupo que atingem maiores latitudes.

2.2 - Espécie ocorrente no Paraguai e Brasil: $A$. kleinii é comum no Rio Grande do Sul e Santa Catarina, diminuindo em expressão em direção à latitude do Trópico de Capricórnio onde parece ser seu limite setentrional. Embora não tenha sido citada por Renvoize (1988) para o Paraná, deve ocorrer nesta região, e ocorre no sul do Paraguai (Figura 19).

2.3 - Espécies exclusivas do Brasil:

a. Espécie de distribuição predominantemente na Região Sul:

A. flaccida ocorre com maior freqüência entre as latitudes $31^{\circ}$ e $25^{\circ} \mathrm{S}$, atingindo porém até a latitude de $21^{\circ} \mathrm{S}$, neste caso ocorrendo em altitudes em torno de $800 \mathrm{~m}$, sendo pouco comum (Figura 20).

b. Espécies com distribuição restrita:

A. ekmaniana, A. riograndensis, Aristida sp. (A. trinii sensu Severo, 1982), e A. teretifolia . A primeira só foi coletada, até o momento, no Paraná; as duas seguintes parecem ser exclusivas do Rio Grande do Sul, onde são pouco freqüentes; a última é pouco comum no Rio Grande do Sul, sendo ainda menos comum em Santa Catarina (Figuras 20-21).

2.4 - Além destes padrões básicos, A. laevis, $A$. circinalis, $A$. venustula, $A$. jubata e $A$. megapotamica var. megapotamica apresentam um padrão intermediário, ocorrendo na área inter e extratropical, incluindo também o Uruguay, Paraguai e Argentina. No Brasil, as duas primeiras ocorrem predominantemente na Região Sul, estendendo-se ao Mato Grosso (Figura 18) e A. venustula no Rio Grande do Sul. A. jubata estende-se, com menor expressão, até Minas Gerais. A última ocorre até o centro e nordeste do país, porém sendo muito menos comum do que na Região Sul (Figuras 19-20).

A. adscensionis L., espécie considerada cosmopolita, apresenta distribuição tropical no Brasil, com limite sul até agora conhecido na Bahia, sendo muito comum na Região Nordeste, onde freqüentemente é confundida com $A$. setifolia. 
De um modo geral, a Província Central e o sul do Brasil são as duas áreas que apresentam uma maior concentração de espécies de Aristida. Na primeira, encontram-se 20 espécies, 7 das quais exclusivas; na segunda, 19 espécies, 5 exclusivas. No norte do Brasil ocorrem 5 espécies, comuns a outras áreas, e na Região Nordeste 8 espécies, apenas uma das quais exclusiva. Convém salientar que o Brasil Central tem sido referido como centro de dispersão de alguns gêneros, como Arthropogon (Gramineae) (Filgueiras 1982). Estes dados vêm ao encontro do que foi referido por Moore (1894), segundo o qual o Brasil Central deve ter sido, em épocas passadas, um centro de diversificação e evolução de espécies.

\section{Conclusões}

O gênero Aristida é dividido em 6 seções, de acordo com a estrutura do lema: Aristida (Chaetaria), Pseudochaetaria, Arthratherum, Pseudarthratherum, Streptachnee e Schizachne.

Com exceção de Pseudochaetaria e Schizachne, as demais estão representadas na América neotropical, das quais somente Aristida e Pseudarthratherum no Brasil, a última com apenas uma espécie, $A$. setifolia $\mathrm{H}$. B. K.

Até o presente foi confirmada a ocorrência de 34 espécies para o Brasil, 13 das quais exclusivas desta área, algumas com distribuição bastante restrita.

Entre os principais caracteres taxonômicos utilizados no gênero, alguns podem ser relacionados com a distribuição das espécies no Brasil. Assim, a posição inversa das glumas aliada à presença de sulco profundo no lema, e a ausência de coluna na arista do lema, são mais comuns nas espécies de distribuição meridional. Por outro lado, o tipo de inflorescência e de calo do lema são caracteres diferenciais decisivos para várias espécies ocorrentes acima do Trópico de Capricórnio, diminuindo sua importância na Região Sul. De um modo geral, as espécies do centro e norte do Brasil apresentam uma descontinuidade morfológica mais acentuada do que as de distribuição meridional, onde algumas são facilmente confundidas no campo.

A maior parte das espécies analisadas é perene, com exceção de $A$. capillacea, $A$. sanctae-luciae e $A$. adscensionis. Da mesma forma, a maior parte ocorre em solos secos e pedregosos, exceto as duas primeiras citadas acima, que ocorrem também em solos brejosos do Brasil Central e dos campos rupestres da Cadeia do Espinhaço.

As espécies estudadas podem ser relacionadas a dois padrões de distribuição geográfica principais: o tropical, que inclui espécies ocorrentes desde a América Central ou norte do Brasil, com limite sul aproximadamente na latitude do Trópico de Capricórnio, e o subtropical incluindo espécies do contingente uruguaio, argentino e sul-brasileiro, a maior parte das quais tem seu limite setentrional de distribuição no Rio Grande do Sul. 


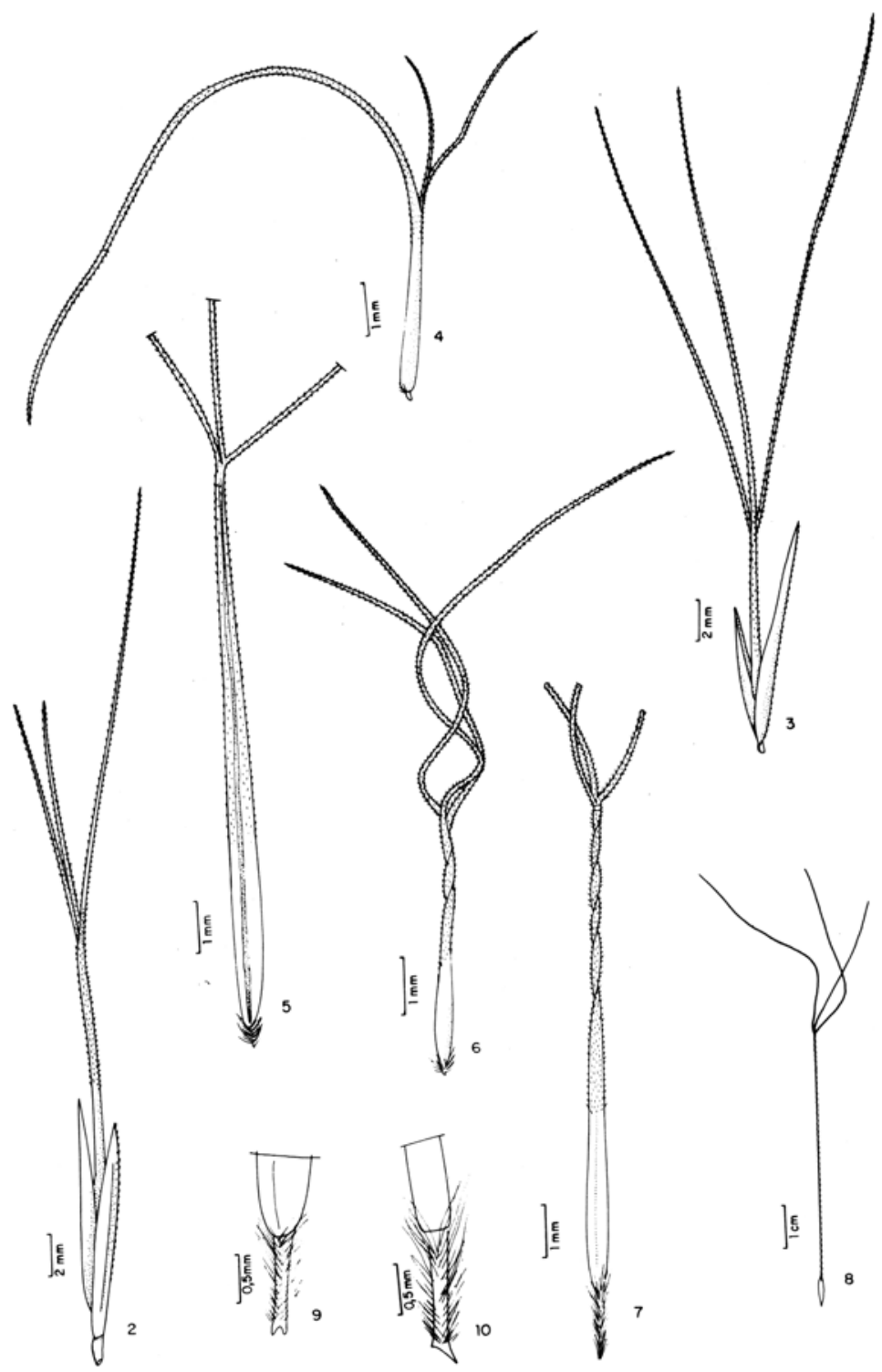

Figuras 2-10 - 2. A. longifolia: espigueta (Burmann 339-SP); 3. A. glaziovii: espigueta (Harley 21109-SPF); 4. A. torta: lema (Martius-M); 5. A. kleinii: lema com sulco ventral; 6. $A$. recurvata: lema, calo obtuso, aristas recurvas (CFSC 8269-SPF); 7. A. setifolia: lema com articulação no ápice da coluna, aristas parcialmente removidas (Pohl 2502-W); 8. A. megapotamica var. megapotamica: antécio (HPF 564); 9. A. riparia: calo do lema bífido (CFCR 2969-SPF); 10. A. megapotamica var. megapotamica: calo do lema (SP 55313). 


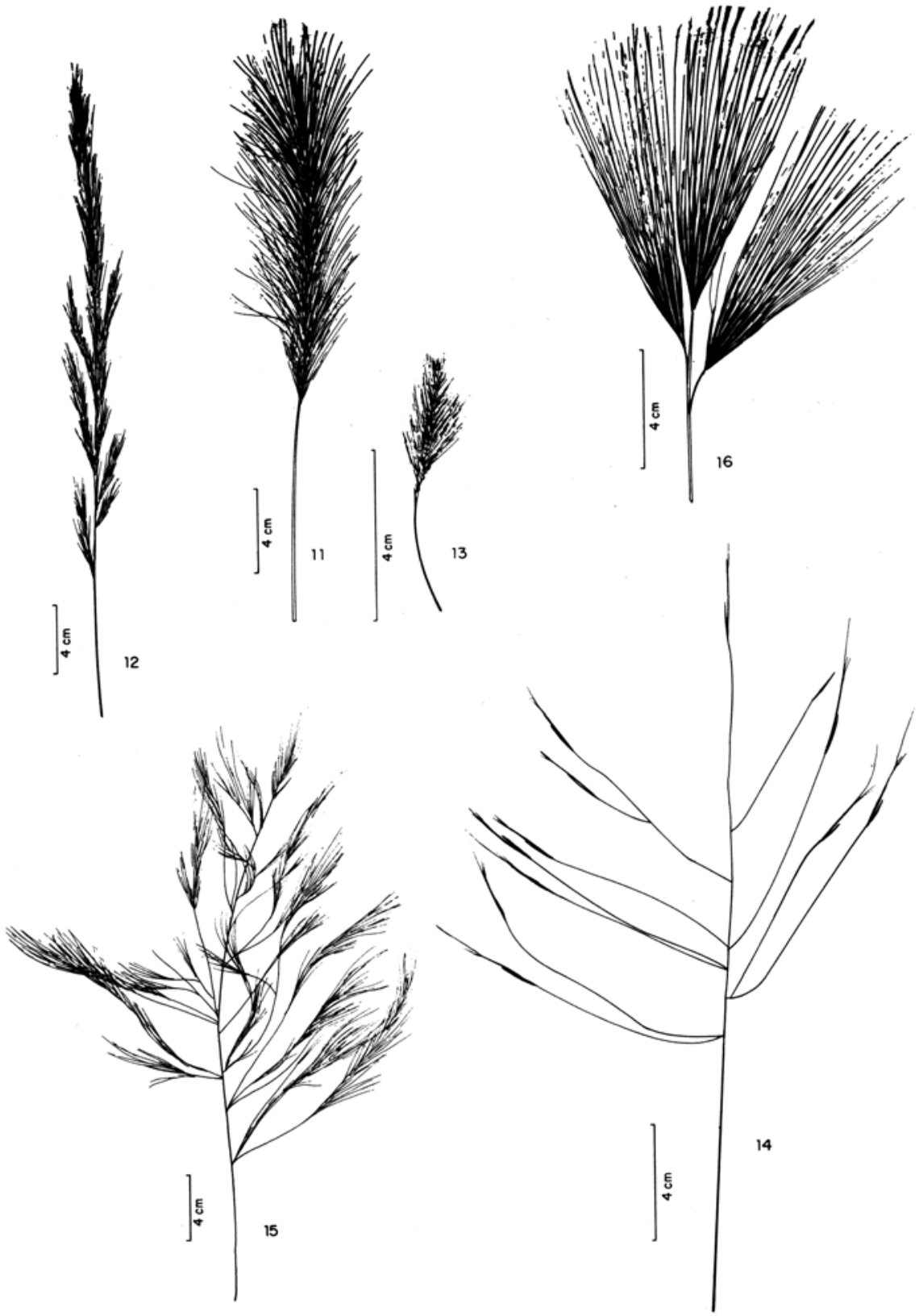

Figuras 11-16 - inflorescências. 11. A. megapotamica var. brevipes (Harıey 15879SPF); 12. A. trinii (CFCR 2970-SPF); 13. A. elliptica (Heringer et al. 770-UB); 14. A. flaccida (Longhi-Wagner 1879-ICN); 15. A. pendula (CFCR 963-SPF); 16. A. murina (Longhi-Wagner 1999-ICN). 


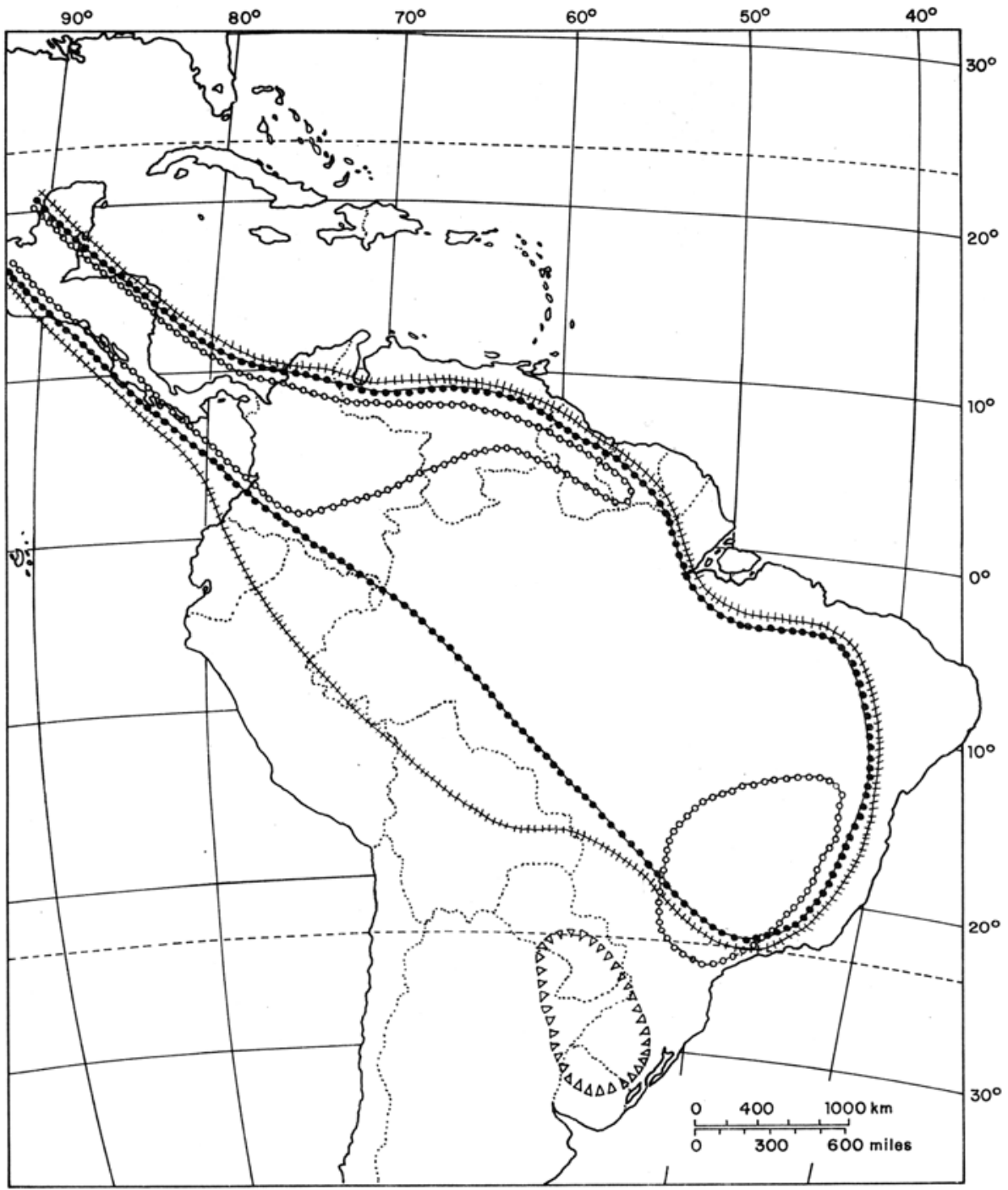

Figura 17 - Distribuição geográfica de: ๑๑ A. longifolia, A. torta; -/-/-/- A. capillacea, A. setifolia; م- $A$. recurvata; $\Delta \Delta \Delta A$. venustula (baseado em mapa Flora Neotropica modificado). 


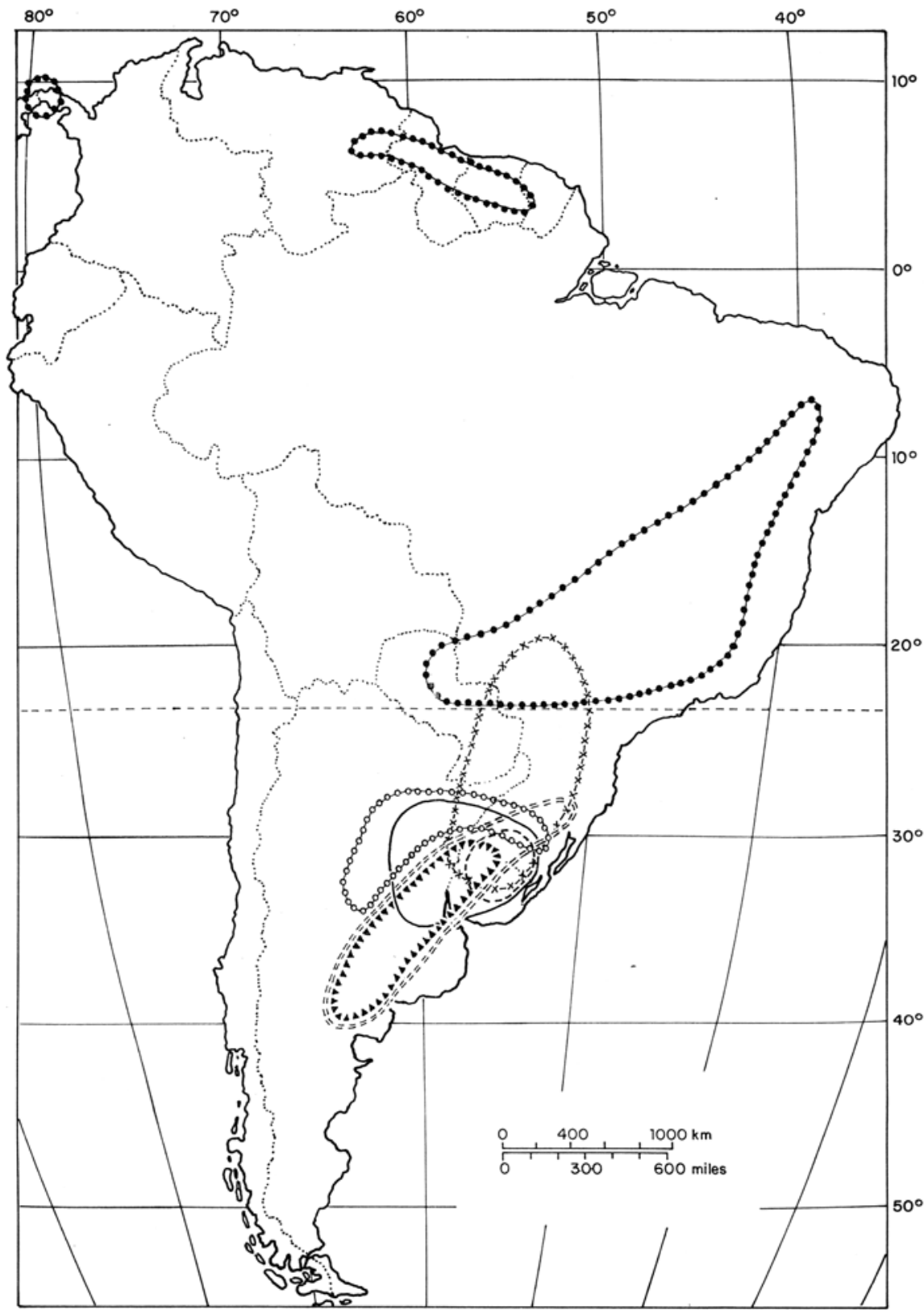

Figura 18 - Distribuição geográfica de: $-\infty$ A. condylifolia, A. venustuloides: $\Delta \Delta \Delta$ A. murina $;===$ A. spegazzinii; - -x-x-A. circinalis, A. laevis; —A. uruguayensis; -- A. echinulata $; \bullet \bullet$ A. riparia (baseado em mapa Flora Neotropica modificado). 


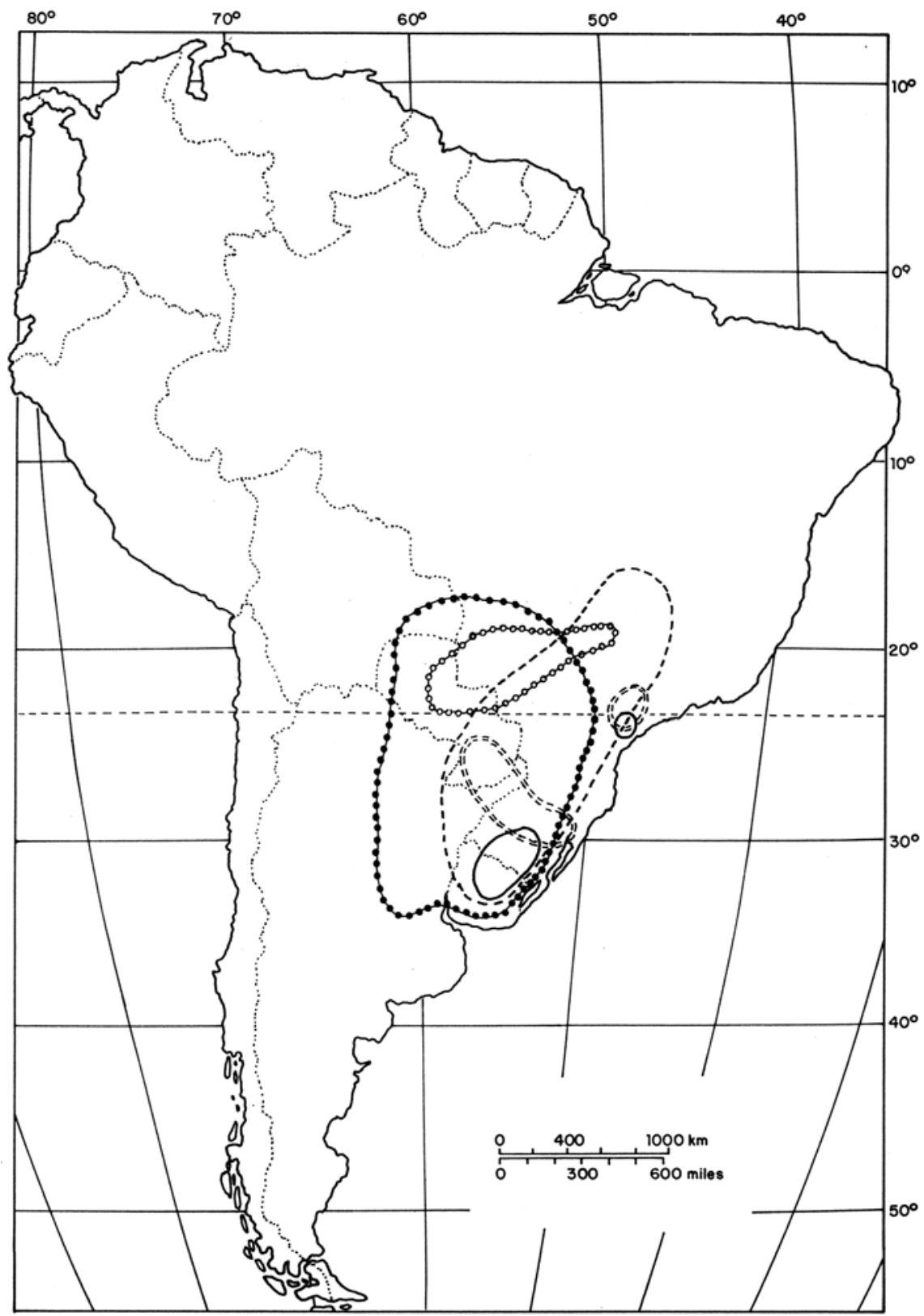

Figura $19-$ Distribuição geográfica de: $\cdots$ A. jubata; - A. filifolia $;==A$. kleinii;,$\circ$ A. oligospira; $\bullet \bullet A$. megapotamica var. megapotamica (baseado em mapa Flora Neotropica modificado). 


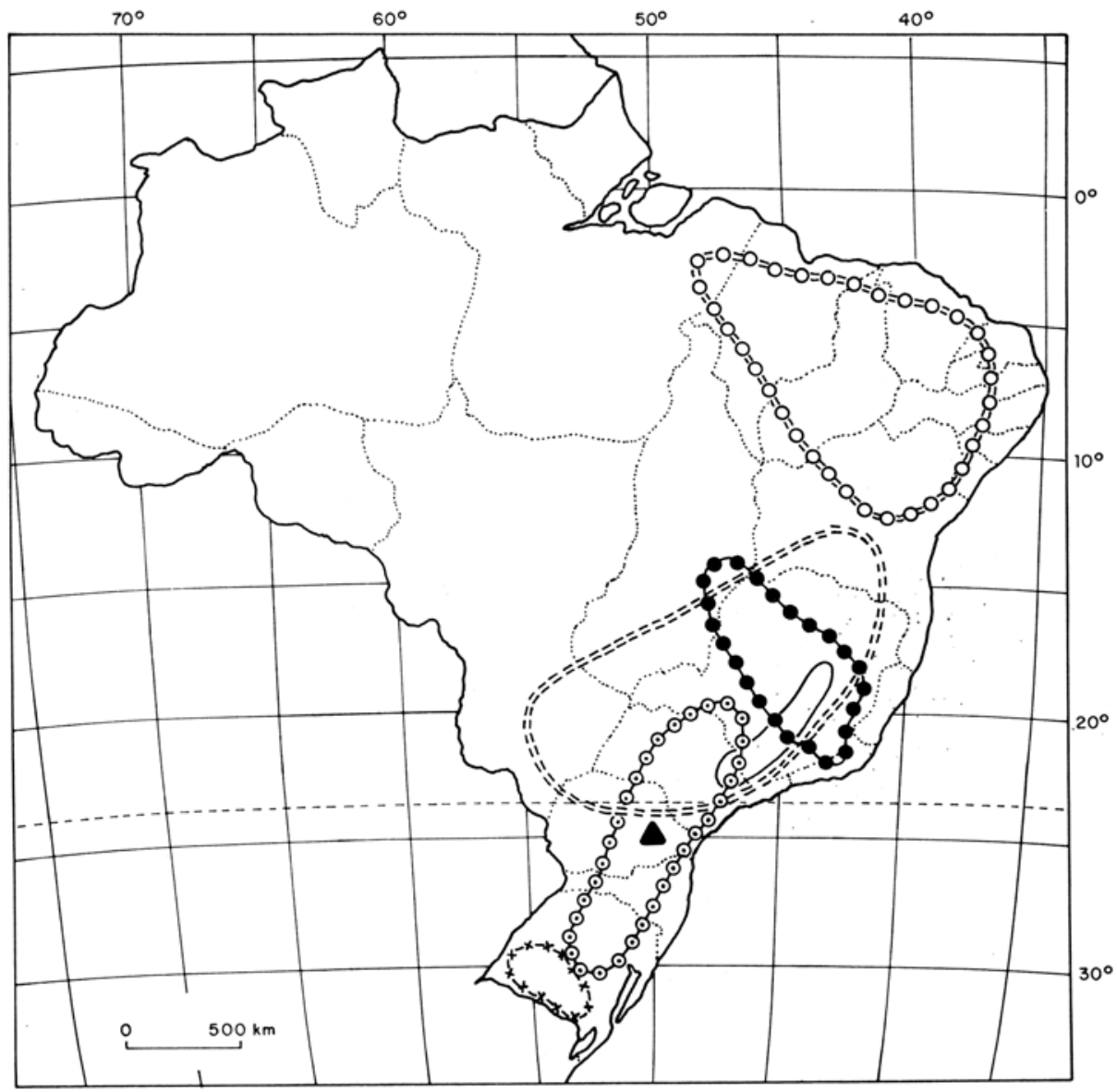

Figura $20-$ Distribuição geográfica de: $-\mathrm{x}-A$. riograndensis e Aristida sp.; $\odot \odot$ A. flaccida $; \Delta$ A. ekmaniana $;===$ A. megapotamica var. brevipes; - A. trinii; $\bullet \bullet$ A. sanctae-luciae; ०ᄋㅇㅇ A. elliptica. 


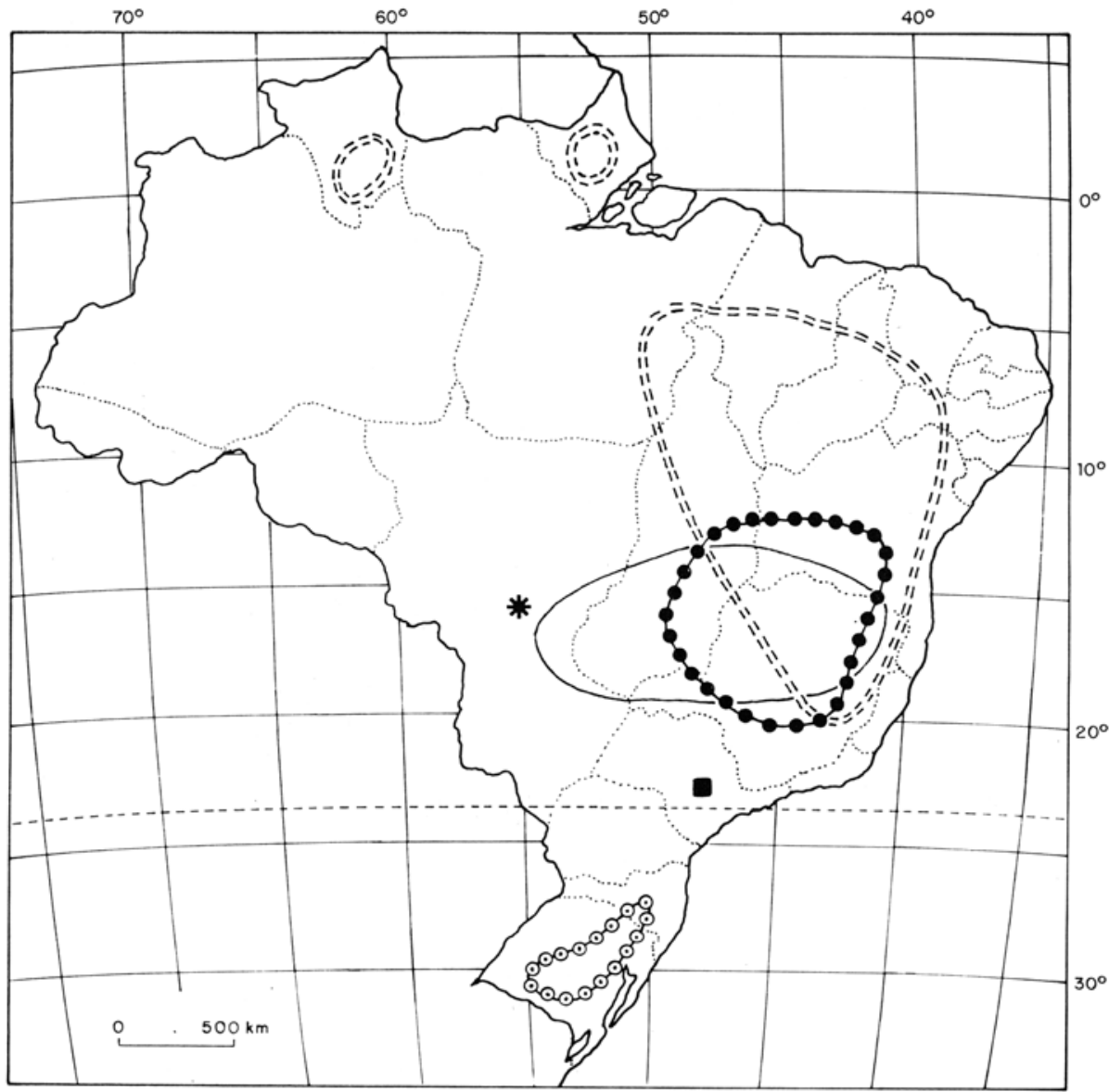

Figura 21 - Distribuição geográfica de: $\odot \odot$ A. teretifolia; - A. succedanea;

$*$ A. chapadensis $;-$ A. pendula $; \bullet$ A. glaziovii $;===$ A. gibbosa . 


\section{Referências bibliográficas}

ANGELY, J. 1965. Flora analítica do Paraná. Curitiba, Phyton.

BENTHAM, G. \& HOOKER, J. D. 1880. Genera Plantarum. Londini, L. Reeve. v. 3 pt. 1.

BROWN, W. V. 1977. The Kranz syndrom and its subtypes in Grass systematics. Mem. Torrey Bot. Club 23(3): 1-97.

BURKART, A. 1969. Flora Ilustrada de Entre Rios (Argentina). Buenos Aires, INTA. t. 6 pt. 2.

BURKART, A. 1975. Evolution of grasses and grasslands in South America. Taxon. 24(1): 53-66.

BURMAN, A. G. 1985. Nature and composition of the grass flora of Brazil. Willdenowia 15: 211-33.

CARO, J. A. 1961. Las especies de Aristida (Gramineae) del centro de la Republica Argentina. Kurtziana 1: 123-206.

CARO, J. A. 1982. Sinopsis taxonomica de las Gramineas argentinas. Dominguezia 4: $1-51$.

CLAYTON, W. D. \& RENVOIZE, S. A. 1986. Genera Graminum. Kew Bulletin additional series XIII.

CLIFFORD, H. T. 1987. Spikelet and floral morphology. In: SODERSTROM, T. R. et al. (eds.). Grass Systematics and evolution. Washington, Smithsonian Institution Press. p. 21-30.

DE WINTER, B. 1965. The South African Stipeae and Aristideae (Gramineae). Bothalia 8(3): 219-404.

DOELL, J. C. (ed.). 1878. Gramineae II. In: MARTIUS, C. F. P. (ed.). Flora Brasiliensis. Monachii, F. Fliescher. v. 2. pt. 3.

EKMAN, E. L. 1913. Die Graser des Brasilianischen Staates Paraná. Arkiv Botanik 13(10): 37-52.

FERNANDES, A. \& BEZERRA, P. 1990. Estudo fitogeográfico do Brasil. Fortaleza, Stylus Com.

FILGUEIRAS, T. S. 1982. Taxonomia e distribuição de Arthropogon Nees (Gramineae). Bradea 3(36): 303-22.

GIULIETTI, A. M. \& PIRANI, J. R. 1988. Patterns of geographic distribution of some plant species from the Espinhaço range, Minas Gerais and Bahia, Brazil. In: HEYER, W. R. \& VANZOLINI, P. E. (eds.). Proceedings of a Workshop on Neotropical distribution patterns. Rio de Janeiro, Academia Brasileira de Ciências. p. 39-69.

HENRARD, J. Th. 1926. A critical revision of the genus Aristida. Med. Rijks Herb. 54: 18-205.

HENRARD, J. Th. 1927. A critical revision of the genus Aristida. Med. Rijks 2(54): 255-428.

HENRARD, J. Th. 1928. A critical revision of the genus Aristida. Med. Rijks Herb. 3(54b): 502-701.

HENRARD, J. Th. 1929. A monograph of the genus Aristida. Med. Rijks Herb. 1(58): 7-131.

HENRARD, J. Th. 1932. A monograph of the genus Aristida. Med. Rijks Herb. 2(58): 157-325. 
HENRARD, J. Th. 1933. A critical revision of the genus Aristida. Med. Rijks Herb. 55c. (suppl.)

HUMBOLDT, F. H. A.; BONPLAND, A. J. \& KUNTH, C. S. 1815. Nova genera et species plantarum. Paris. v. 1. Reprint 1963, J. Cramer.

LONGHI-WAGNER, H. M. 1986. A subfamília Chloridoideae (Gramineae) na Cadeia do Espinhaço, Brasil. Tese de Doutoradó.'São Paulo, Inst. Bioc., Univ. São Paulo. (não publicado).

MOORE, S. M. 1894-1896. The phanerogamic Botany of the Matto Grosso Expedition 1891-92. In: The transactions of the Linnean Society of London. The Linnean Society. London. (sér. 2, v. 4-Botany).

NEES, C. G. 1829. Agrostologia brasiliensis. Stuttgartie et Tübingen, J. G. Cottae. v. 2. NICORA, E. G. 1978. Flora Patagonica. pt. 3 Gramineae. Buenos Aires, INTA.

PARODI, L. 1961. La taxonomia de las gramineas argentinas a la luz de las investigaciones más recentes. In: Recent Advances in Botany. Toronto, Univ. Toronto. v. 1. p. 125-30.

RENVOIZE, S. A. 1984. The Grasses of Bahia. Kew, Royal Botanic Gardens.

RENVOIZE, S. A. 1988. Hatschbach's Paraná Grasses. Kew, Royal Botanic Gardens. SEVERO, B. A. 1982. O gênero Aristida L. (Gramineae) no Rio Grande do Sul. Tese de Mestrado. Porto Alegre, Univ. Federal do Rio Grande do Sul (não publicado).

SMITH, L. B.; WASSHAUSEN, D. C. \& KLEIN, R. M. 1981. Gramíneas. Flora Ilustrada Catarinense (Gram.): 1-435.

STEBBINS, G. I. \& CRAMPTON, B. 1961. A suggested review of the grass genera of temperate North America. In: Recent Advances in Botany. Toronto, Univ. Toronto. v. 1. p. 133-44.

TATEOKA, 1957. Miscellaneous papers on the Phylogeny of Poaceae. Proposition of a new phylogenetic system of Poaceae. Journal jap. Bot. 32(9): 275-87.

TRINIUS, C. B. \& RUPRECHT, F. J. 1842. Species Graminum. Mem. Acad. Inst. St. Peters., sér. b, 5: 1-189.

VALLS, J. F. M. 1980. Gramíneas nativas e sua importância forrageira: situação do estudo no Brasil. In: CONGRESSO NACIONAL DE BOTÂNICA, 30, Campo Grande, Simpósio. Brasília, EMBRAPA, 1980, p. 7-23 (Plantas forrageiras, EMBRAPA-CENARGEN, doc. 1.). 